\title{
Adhesion resistance of nanocoatings of tool working surfaces
}

\author{
Larissa Frolenkova, Alexander Tarapanov, Michael Selemenev, Alexander Novikov and \\ Elena Selemeneva
}

Oryol State University named after I.S. Turgenev, Oryol, Russia

\begin{abstract}
To increase the strength, reliability, durability of the cutting tool, various wear-resistant coatings are applied to its cutting part. At the same time, under cutting conditions, it is necessary to ensure a high degree of adhesion of the coating to this part of the tool. The paper presents theoretical aspects that make it possible to select the operating modes of the tool that preserve the integrity of the "coating-base" system.
\end{abstract}

\section{Introduction}

The main cause of cutting tool wear is significant frictional forces between the working surface and the workpiece material. With an increase in the axial cutting force, the lubricant does not penetrate between the rubbing side surfaces of the profile, and then dry friction arises, which increases the cutting temperature. Due to elastic deformations and irregularities in processing, the threads tend to jam in the grooves of the tool, which also increases friction, and sometimes leads to welding of rubbing surfaces and tool breakage.

The surface energy of solids is high - about $500-5000 \mathrm{mD} / \mathrm{m}^{2}$, and the surface tension of liquids is 10-100 times less. Therefore, to prevent the spreading of liquids over a solid, it is necessary to reduce the surface tension of the solid by placing between the liquid and the solid some kind of interlayer - a film, the surface tension of which is close to the surface energy of the liquid. The application of films of surfactants (surfactants) to reduce the spreading of lubricating oils was first proposed by the French scientist Vug in the 20s of last century[1, 2].

Epilamination is the process of deposition of surfactants onto a hard surface. The nature of adsorption and the orientation of the deposited molecules are determined by the interaction of the adsorption centers of the surface with the active centers of the molecules. The spatial arrangement of adsorbed molecules depends on their polarity and deposition conditions, can be both vertical and horizontal, and can also form any angle with a solid surface [3].

Epilamination involves the formation of multimolecular layers with regularly oriented molecules on the surface of a solid. The degree of orientation and the thickness of the oriented layer depend on the nature of the solid and surfactant molecules. Thus, within a homologous series of fatty acids, the degree of orientation increases with an increase in the chain length of the molecule [5]. 


\section{Theory}

To assess the strength of an adhesive joint during shear of the coating relative to the base, the condition of equality of the energy of elastic deformations in the shear coating of the energy of its adhesion is applied [4]. Knowing the energy of adhesion and sufficiently accurately expressing the energy of elastic deformations through the acting classical stresses, one can estimate their maximum permissible value, which does not lead to separation or shear of the coating. The paper shows that the classical approach based on this idea can lead to erroneous results. Therefore, to describe the elastic properties of the coating, it is proposed to use the micropolar theory of elasticity. The expression for determining the adhesion energy is:

$$
\frac{h \tau_{\max }^{2}}{2 \mu}=F_{a},
$$

where is the $\tau_{\max }$ - maximum permissible shear stress, $\mathrm{h}$ - is the thickness of the coating; $\mu$ - Lame parameter; $F_{a}$ - the energy of adhesion.

The surface of the tool, having a metal base, has an orienting effect due to the presence of a near-surface electric field with an intensity $E \sim 10^{9} \mathrm{~V} / \mathrm{m}$ - for each specific material, this value can be estimated by physical methods $[6,7]$. When connecting the coating material to the base, its atoms or molecules continue the atomic lattice, the base structure. In this case, they acquire an orientation that is not characteristic of their orientation in the structure of the material, far from the surface of the adhesive contact. , performing work against the internal forces and moments of the coating material.wThis is equivalent to the fact that the same atoms turn out to be rotated by the orienting field of the base material and distributed along the contact surface by moments at a certain angle of elastic deformations is concentrated, which is not taken into account when constructing expression (1). $\Delta$ Because of this, in this material, even in the absence of external influences in the surface layer, a stressed state develops, a certain energy. Given these reasons, the dependence of the form:

$$
\frac{h \tau_{\max }^{2}}{2 \mu}+\Delta=F_{a} .
$$

where is the $\tau_{\max }$ - maximum permissible shear stress, $\mathrm{h}$ - is the thickness of the coating; $\mu$-Lame parameter; $F_{a}$ - the energy of adhesion, $\Delta$ - elastic energy.

Then, when estimating the permissible shear stress $\tau_{\max }$, it turns out that the estimate

$$
\tau_{\text {max }}^{*}=\sqrt{\frac{2 \mu\left(F_{a}-\Delta\right)}{h}}<\tau_{\text {max }}=\sqrt{\frac{2 \mu F_{a}}{h}} .
$$

This inequality indicates that the calculation based on classical concepts of the elastic properties of the surface layer of the material leads to overestimated values $\mu$ and $\Delta$ of the permissible shear stresses, it is proposed to use the micropolar theory of elasticity [8]. It is based on the idea that a particle of a continuous elastic medium has not three, as in the classical case, but six degrees of freedom. Accordingly, to describe its motion, a displacement vector and a vector of a small angle of rotation $\vec{\omega}(\vec{r})(\vec{r}-$ is the radius vector of the particle center of mass) are used. To estimate the quantities

The expression for calculating the bulk density of the energy of elastic deformations $w$ according to this model can be represented in the form [9]:

$$
w=\frac{\mu+\alpha}{2} \gamma_{i j} \gamma_{i j}+\frac{\mu-\alpha}{2} \gamma_{i j} \gamma_{j i}+\frac{\lambda}{2} \gamma_{k k} \gamma_{n n}+\frac{\gamma+\varepsilon}{2} \kappa_{i j} \kappa_{i j}+\frac{\gamma-\varepsilon}{2} \kappa_{i j} \kappa_{j i}+\frac{\beta}{2} \kappa_{k k} \kappa_{n n},
$$


Where $\lambda, \mu$-classical constants, Lamé coefficients; $\alpha, \beta, \gamma, \varepsilon-$ are additional constants that need experimental or theoretical (based on physical considerations) definition; $\gamma_{i j}=u_{i, j}-\epsilon_{k j i} \omega_{k} ; \kappa_{i j}=\omega_{i, j}$ - tensors of material deformations $\left(\epsilon_{k j i}-\right.$ three-index LeviCivita symbol; the subscript after the decimal point means differentiation along the coordinate with the corresponding number; $u_{i}, \omega_{i}-$ components of vectors $\vec{u}, \vec{\omega}$ in the orthonormal basis $\vec{e}_{i}$ of the Cartesian coordinate system $x_{i}$ ). In expression (3) summation is performed over a repeated index.

Taking into account that internal stresses $\sigma_{i j}$ and moments $\mu_{i j}$ at constant temperature are expressed through strain tensors using the relations

$$
\begin{gathered}
\sigma_{i j}=\frac{\partial w}{\partial \gamma_{i j}}=2 \mu \gamma_{(i j)}+2 \alpha \gamma_{\langle i j\rangle}+\lambda \gamma_{k k} \delta_{i j}, \\
\mu_{i j}=\frac{\partial w}{\partial \kappa_{i j}}=2 \gamma \kappa_{(i j)}+2 \varepsilon \kappa_{\langle i j\rangle}+\beta \kappa_{k k} \delta_{i j},
\end{gathered}
$$

for the bulk density of the energy of elastic deformations w, one can obtain the expression:

$$
w=\mu^{*} \sigma_{(i j)} \sigma_{(i j)}+\alpha^{*} \sigma_{\langle i j\rangle} \sigma_{\langle i j\rangle}+\frac{\lambda^{*}}{2} \sigma_{k k} \sigma_{n n}+\gamma^{*} \mu_{(i j)} \mu_{(i j)}+\varepsilon^{*} \mu_{\langle i j\rangle} \mu_{\langle i j\rangle}+\frac{\beta^{*}}{2} \mu_{k k} \mu_{n n} .
$$

In expressions (4) - (6), the parentheses mean the symmetric part, and the angular ones the antisymmetric part of the corresponding tensor; $\delta_{i j}$ - the Kronecker symbol;

$$
\begin{gathered}
\mu^{*}=\frac{1}{4 \mu} ; \quad \alpha^{*}=\frac{1}{4 \alpha} ; \quad \gamma^{*}=\frac{1}{4 \gamma} ; \quad \varepsilon^{*}=\frac{1}{4 \varepsilon} ; \\
\lambda^{*}=-\frac{\lambda}{6 \mu K} ; \quad \beta^{*}=-\frac{\beta}{6 \gamma \Omega} ; \quad K=\lambda+\frac{2}{3} \mu ; \quad \Omega=\beta+\frac{2}{3} \gamma .
\end{gathered}
$$

In expression (6), the first term fully corresponds to the classical concepts of the volumetric energy density of elastic deformations. Further, when assessing the role of the sum of the remaining terms, we assume that the stresses $\sigma_{(i j)}$ coincide with their classical values.

In the case of a plane shear, which is of interest when considering the problem discussed in this work, the vectors of displacements and microrotations take the form:

$$
\vec{u}=\left(u_{1}\left(x_{2}\right), 0,0\right) ; \quad \vec{\omega}=\left(0,0, \omega_{3}\left(x_{2}\right)\right) \text {. }
$$

In this case, the axis $O x_{1}$ is directed along the contact plane of the coating and the base in the direction of the parallel shear stress $\tau \equiv \tau_{1}$. The axis $O x_{2}$ is directed perpendicular to the contact plane. The shift occurs in the plane $x_{1} O x_{2}$. As a result, only the following quantities are considered:

$$
\begin{array}{cc}
\gamma_{12}=u_{1,2}+\omega_{3} ; & \kappa_{32}=\omega_{3,2} ; \\
\gamma_{(12)}=\frac{1}{2} u_{1,2} ; & \gamma_{\langle 12\rangle}=\frac{1}{2} u_{1,2}+\omega_{3} ; \\
\kappa_{(32)}=\frac{1}{2} \omega_{3,2} ; & \kappa_{\langle 23\rangle}=\frac{1}{2} \omega_{3,2} .
\end{array}
$$

The equilibrium equations in stresses within the framework of the considered model of the medium have, in the general case, the form:

$$
\sigma_{i j, i}=0 ; \quad \mu_{i j, i}+\in_{i j k} \sigma_{i k}=0 .
$$

In the considered plane case of pure shear, based on (11), having written down the 
second equation in displacements, one can obtain:

$$
\sigma_{21,2}=0 ; \quad(\gamma+\varepsilon) \omega_{3,22}+2 \alpha u_{1,2}-4 \alpha \omega_{3}=0 .
$$

The boundary conditions for them are the relations:

$$
\sigma_{21}(h)=\tau ; \mu_{23}(h)=0 ; u_{1}(0)=0 ; \omega_{3}(0)=\omega^{0}
$$

The first equation of system (12), taking into account the first of the boundary conditions (13), gives reason to believe that, as in the case of using the classical model of a linearly elastic medium, within the framework of the considered model, the shear stress that shears the coating is uniformly distributed over its thickness $h$ and is equal to the acting shear stress on the surface.

$$
\sigma_{21}=\tau \text {. }
$$

This result, as well as relations (4) and (5), connecting internal stresses and strains, and the last of relations (13), allow us to conclude that the distributions of displacements $u_{1}\left(x_{2}\right)$ and rotations $\omega_{3}\left(x_{2}\right)$, as well as their derivatives over the coating thickness, are nonzero. Based on (6) and (7), taking into account (5), this conclusion leads to the statement that the elastic deformation energy $\mathrm{W}$, concentrated under the unit of the surface area of the coating, can be written in the form:

$$
W=\frac{h \tau^{2}}{2 \mu}+\frac{\alpha}{2} \int_{0}^{h}\left(\frac{1}{2} u_{1,2}\left(x_{2}\right)+\omega_{3}\left(x_{2}\right)\right)^{2} d x_{2}+\frac{\gamma+\varepsilon}{2} \int_{0}^{h}\left(\omega_{3,2}\left(x_{2}\right)\right)^{2} d x_{2} .
$$

Comparing it with the left-hand side of equality (3), one can see that

$$
\Delta=\frac{\alpha}{2} \int_{0}^{h}\left(\frac{1}{2} u_{1,2}\left(x_{2}\right)+\omega_{3}\left(x_{2}\right)\right)^{2} d x_{2}+\frac{\gamma+\varepsilon}{2} \int_{0}^{h}\left(\omega_{3,2}\left(x_{2}\right)\right)^{2} d x_{2} .
$$

It can be seen that the classical estimate of the adhesion energy is lower than that which can be done by equating (15) to the value $F_{a}$. Obviously, for sufficiently large values $\alpha$ and $\gamma+\varepsilon$ classical estimates $\tau_{\max }$ cannot be used.

In work [10], the following estimates $E \sim 10^{9} \mathrm{~V} / \mathrm{m}$ were made for the tension values: $\alpha \approx 10^{8} \mathrm{~N} / \mathrm{m}^{2} ; \gamma+\varepsilon \approx 10^{6} \mathrm{~J} / \mathrm{m}$. For a coating with a thickness $h \approx 10^{-4} \mathrm{~m}, u_{1} \approx 10^{-6} \mathrm{~m}$, $\omega \approx 10^{-3}$ radian the value of $\Delta$ may turn out to be comparable with a value $F_{a}$, of the order of $1 \mathrm{~J} / \mathrm{m}^{2}$. (When evaluating the value of $\Delta$ it was taken into account that the integrand has the order of its mean value, equal to the half-sum of its extreme values, and the derivative is the ratio of the mean value to the coating thickness). Therefore, the calculation $\tau_{\max }$ based on classical concepts can lead to significant errors. This confirms the need to take into account the above views when calculating cutting conditions for tools with coatings.

\section{Findings}

The article presents a theoretical justification for the need to use, when assessing the value of the maximum permissible shear stresses in the coatings of cutting tools, the concepts of the elastic properties of materials used by the micropolar theory of elasticity. The given numerical estimates indicate the need for a more accurate physical and theoretical, based on the micropolar theory of elasticity, an analysis of the situation considered in the work and the development of recommendations necessary for practical applications. 


\section{References}

1. Vitcovsky I. V., Konev A. N., Shorkin V. S., Kzaev N. D., Rusanov A. E., Khoroshikh V. M. and Leonov S. L. Adhesion energy estimation of some composite materials // Plasma Devices and Operations. - 2003. - V. 11(2). - P. 81 - 87.

2. Shorkin VS, Frolenkova L. Yu., Azarov AS Taking into account the influence of the triple interaction of medium particles on the surface and adhesion properties of solids // Materials Science. - 2011. - No. 2. - P. 2 - 7.

3. Vitkovsky I. V., Konev A. N., Shorkin V. S., Yakushina S. I. Theoretical estimation of the discontinuity of the adhesive contact of the elements of the liquid metal blanket of a thermonuclear reactor // Journal of technical physics, 2007. - V. 77. - Issue 6. - p. 28 - 33.

4. Shorkin V.S., Batishchev A.N., Kuznetsov Yu.A. Analysis of the stress state of wearresistant coatings of restored parts // Mechanization and electrification of agriculture, 2003. -No. 3. - P. 28-30.

5. Zimon AD Adhesion of films and coatings. - M .: Chemistry, $1977.352 \mathrm{p}$.

6. Partensky MB Self-consistent electronic theory of a metallic surface // Uspekhi fizicheskikh nauk. - 1979. - T. 128. - Issue. 1. - S. 69 - 106.

7. Vakilov AN, Mamonova MV, Prudnikov VV Adhesion of metals and semiconductors in the framework of dielectric formalism // Solid State Physics. - 1997. - T. 39. - No. 6. - P. $964-967$.

8. Kuvshinskii EV, Aero EL Continual theory of asymmetric elasticity. Taking into account internal rotation // FTT. - 1963. -T. 5.- Iss. 9. -C. 251 - 264.

9. Novatsky V. Theory of elasticity. - M .: Mir, 1975 .-- 872 p.

10. Romashin SN, Sedov AV, Khoroshilova MV, Shorkin VS Influence of the electrostatic field on the mechanical properties of a dielectric // Fundamental and applied problems of technology and technology. - 2012. - No. 1 (291). - S. 3 - 9. 